\title{
Tratamento das lesões do ligamento cruzado anterior em jogadores profissionais de futebol por cirurgiões ortopedistas*
}

\section{Treatment of Anterior Cruciate Ligament Injuries in Professional Soccer Players by Orthopedic Surgeons}

\author{
Gustavo Gonçalves Arliani ${ }^{1}$ Vitor Luis Pereira ${ }^{20}$ Renan Gonçalves Leão ${ }^{2}$ Paulo Schmidt Lara ${ }^{1}$ \\ Benno Ejnisman ${ }^{1}$ Moisés Cohen ${ }^{1}$ \\ ${ }^{1}$ Departamento de Ortopedia e Traumatologia, Centro de \\ Traumatologia do Esporte (CETE), Escola Paulista de Medicina, \\ Universidade Federal de São Paulo, SP, Brasil

\begin{abstract}
Endereço para correspondência Vitor Luis Pereira, Rua Kiel 55, apto. 3B - Casa Verde, São Paulo, SP, CEP: 02512-050, Brasil (e-mail: vitor_luis_pereira@yahoo.com.br).
\end{abstract}

2 Departamento de Ortopedia e Traumatologia, Escola Paulista de Medicina, Universidade Federal de São Paulo, SP, Brasil

Rev Bras Ortop 2019;54:703-708.

\begin{abstract}
Resumo
Objetivo Descrever o tratamento realizado por especialistas das lesões do ligamento cruzado anterior (LCA) em jogadores profissionais de futebol.

Métodos Estudo transversal, no qual ortopedistas vinculados a clubes participantes do Campeonato Brasileiro de Futebol responderam a um questionário sobre o tratamento das lesões do LCA em jogadores profissionais de futebol.

Resultados Os especialistas aguardam entre uma e quatro semanas após a lesão do LCA para realizar o tratamento cirúrgico. Utilizam técnica com incisão e banda únicas auxiliada por artroscopia, perfuração do túnel femoral via portal acessório medial, e autoenxerto quádruplo de tendões flexores ou autoenxerto de tendão patelar. Os jogadores são liberados para correr em linha reta após três a quatro meses; para exercícios com bola sem contato com outros atletas, após quatro a seis meses; e o retorno ao esporte acorre após seis a oito meses. O principal parâmetro usado para o

Palavras-chave

- ligamento cruzado anterior

- joelho/cirurgia

- futebol

- reabilitação retorno ao esporte é o teste de força isocinético. Os especialistas estimam que mais de $90 \%$ dos jogadores operados por lesão do LCA retornam ao esporte profissional, e entre $60 \%$ e $90 \%$ retornam com o mesmo nível ou com um nível melhor de desempenho. Conclusão Este estudo descreve de forma satisfatória as principais práticas cirúrgicas e pós-operatórias adotadas pelos especialistas nessa população altamente específica de pacientes.
\end{abstract}

\footnotetext{
Trabalho realizado no Centro de Traumatologia do Esporte (CETE), Departamento de Ortopedia e Traumatologia, Escola Paulista de Medicina, Universidade Federal de São Paulo, SP, Brasil.
}

recebido

20 de Abril de 2018

aceito

06 de Agosto de 2018
DOI https://doi.org/

10.1055/s-0039-1697017. ISSN 0102-3616.
Copyright $\odot 2019$ by Sociedade Brasileira License terms de Ortopedia e Traumatologia. Published by Thieme Revinter Publicações Ltda, Rio de Janeiro, Brazil 


\begin{abstract}
Keywords

- anterior cruciate ligament

- knee/surgery

- soccer

- rehabilitation

Objective To describe the treatment provided by specialists for anterior cruciate ligament $(\mathrm{ACL})$ lesions in professional soccer players.

Methods A cross-sectional study in which orthopedic surgeons affiliated to soccer teams competing in the Brazilian Soccer Championship answered a questionnaire about the treatment of $\mathrm{ACL}$ injuries in professional soccer players.

Results The specialists wait between one to four weeks after the ACL injury to perform the surgical treatment. They use a single incision and single-bundle reconstruction, assisted by arthroscopy, femoral tunnel drilling by an accessory medial portal, and quadruple flexor tendon autografts or patellar tendon autografts. After three to four months, the players are allowed to run in a straight line; after four to six months, they begin to practice exercises with the ball without contact with other athletes; and, after six to eight months, they return to play. The main parameter used to determine the return to play is the isokinetic force test. The specialists estimate that more than $90 \%$ of elite soccer players return to playing professionally after an ACL reconstruction, and 60 to $90 \%$ return to play at their prior or at a greater level of performance.

Conclusion The present article successfully describes the main surgical practice and post-surgery management adopted by specialists in this highly-specific population of patients.
\end{abstract}

\section{Introdução}

O futebol é o esporte mais popular do mundo, com mais de 265 milhões de jogadores ativos em todo o planeta, ${ }^{1}$ e é o principal causador de lesões esportivas. ${ }^{2}$ Uma das lesões do joelho mais comuns é a ruptura do ligamento cruzado anterior (LCA), ${ }^{3-6}$ lesão grave que requer predominantemente tratamento cirúrgico. ${ }^{7,8}$ Embora o risco de sofrer essa lesão seja baixo na população geral, é consideravelmente maior nos praticantes de esportes coletivos. ${ }^{9}$

No futebol profissional, essa lesão tem consequências econômicas importantes; o afastamento dos treinos e das competições por meses, associado a questões como cumprimento de contratos, agendas, compromissos de publicidade e outros frequentemente implica enorme pressão para o retorno rápido ao esporte.

O objetivo primário da reconstrução ligamentar nesses pacientes consiste em restaurar a função fisiológica do joelho lesado e permitir ao atleta retorno ao futebol o quanto antes e no mesmo nível anterior ao da lesão, além de auxiliar a prevenir processos degenerativos que poderiam ocorrer. ${ }^{10,11}$ Não há consenso quanto ao melhor tratamento, tampouco quanto ao tempo necessário para reabilitação e retorno seguro às atividades competitivas. ${ }^{9,10,12}$ Diante da ausência de evidências sólidas, a experiência dos especialistas é um fator importante na condução desses casos. Nosso objetivo consiste na descrição do tratamento das lesões do LCA em atletas profissionais de futebol realizado por ortopedistas vinculados a clubes do Campeonato Brasileiro de Futebol.

\section{Materiais e Métodos}

Este estudo transversal foi aprovado pelo Comitê de Ética em Pesquisa da Universidade Federal de São Paulo no parecer de número 2.221.990. Foi desenvolvido a partir da entrevista com
61 ortopedistas vinculados a clubes brasileiros de futebol profissional, sendo majoritariamente clubes participantes do Campeonato Brasileiro de Futebol. Os especialistas foram abordados individualmente pelos integrantes da equipe de pesquisa, por meio de contato telefônico ou via endereço eletrônico. Todos foram submetidos a um questionário de múltipla escolha contendo 17 questões, após assinatura do termo de consentimento livre e esclarecido. 0 questionário foi baseado em modelos utilizados em pesquisas internacionais, $\mathrm{e}$ foi adaptado pelos autores, tendo seu conteúdo aprovado por comissão de médicos especialistas na área.

As questões foram desenvolvidas com o objetivo de obter dados sobre o perfil dos entrevistados e sua experiência, métodos de tratamento, técnicas cirúrgicas e práticas pósoperatórias.

Foram utilizados os softwares Statistical Package for the Social Sciences (SPSS, IBM Corp., Armonk, NY, EUA), versão20.0, Minitab (Minitab, LLC, State College, PA, EUA), versão 16, e Excel Office 2010 (Microsoft Corp., Redmond, WA, EUA). Para as variáveis quantitativas, foi realizada análise descritiva completa. Obtivemos baixa variabilidade nas respostas (coeficiente de variação [CV] menor do que $50 \%$ ), o que demonstra dados homogêneos. As variáveis qualitativas foram analisadas por meio do cálculo de frequências absolutas e relativas, testes de igualdade de proporções, análise de intervalos de confiança (ICs) estatística de $95 \%$, e valor de $p$ (erro estatístico admitido) definido como menor ou igual a 0,05 . Os dados obtidos foram comparados aos encontrados na literatura mundial. Todos os resultados diferentes dos parâmetros estabelecidos foram detalhados.

\section{Resultados}

De um total de 61 especialistas participantes, 31 eram vinculados a clubes pertencentes à Série $\mathrm{A}$ do campeonato 
Tabela 1 Análise descritiva completa das variáveis quantitativas das questões 1, 2 e 3

\begin{tabular}{|l|l|l|l|l|l|l|l|l|l|l|}
\hline Descritor & Média & Mediana & Desvio padrão & CV & Q1 & Q3 & Mín. & Máx. & N & IC \\
\hline Idade (anos) & 47,43 & 46 & 8,63 & $18 \%$ & 42 & 53 & 30 & 65 & 61 & 2,17 \\
\hline Tempo de formado (anos) & 23,64 & 24 & 8,56 & $36 \%$ & 18 & 30 & 6 & 41 & 61 & 2,15 \\
\hline Atuação na ortopedia (anos) & 20,33 & 19 & 8,89 & $44 \%$ & 15 & 27 & 1 & 36 & 61 & 2,23 \\
\hline
\end{tabular}

Abreviaturas: CV, coeficiente de variação; IC, intervalo de confiança; Máx., máximo; Mín., mínimo; N, número; Q, questão.

brasileiro de futebol (50,8\% dos entrevistados), 21 eram vinculados a clubes da Série B (34,4\%), e 9 eram vinculados a clubes da Série $C / D$ ou a clubes que disputam campeonatos regionais de futebol (14,8\%). A - Tabela 1 descreve as informações obtidas nas três primeiras questões.

Quanto à cirurgia definitiva após lesão do LCA, 45,9\% dos entrevistados aguardam de 1 a 2 semanas para sua realização diante de uma lesão aguda e isolada do LCA; 34,4\% aguardam entre 2 e 3 semanas; 16,4\% realizam cirurgia imediata; e apenas 3,3\% aguardam entre 4 e 6 semanas para a abordagem definitiva. Estatisticamente, não houve diferença entre as opções de espera de 1 a 2 semanas e de 2 a 3 semanas $(p=0,196)$.

A técnica cirúrgica preferencialmente escolhida foi a de incisão e banda únicas auxiliada por artroscopia para $78,7 \%$, seguida de incisão dupla e banda única auxiliada por artroscopia para $16,4 \%$, e por incisão única e banda dupla auxiliada por artroscopia para $4,9 \%$. Nenhum entrevistado optou por outras alternativas.

A perfuração do túnel femoral via portal acessório medial (transportal) foi a escolha de 50,8\% dos entrevistados; $24,6 \%$ optaram pela técnica outside-in, $23 \%$ optaram pela transtibial, e apenas $1,6 \%$, pela dupla incisão.

O autoenxerto mais usado foi o quádruplo de tendões flexores por $49,2 \%$ dos entrevistados, seguido pelo autoenxerto de tendão patelar por $34,4 \%$. Em terceiro lugar, o autoenxerto quíntuplo ou sêxtuplo de tendões flexores, com $13,1 \%$, e, por fim, o autoenxerto do tendão quadricipital, com 3.3\%. Não é possível afirmar que exista diferença entre as duas opções mais adotadas ( $p=0,099)$. As - Figuras 1 e 2 descrevem as principais preocupações citadas pelos entrevistados, de acordo com o autoenxerto escolhido.

O movimento passivo contínuo (MPC) é usado na reabilitação após reconstrução do LCA por $67,2 \%$ dos ortopedistas. A corrida em linha reta foi liberada entre 3 e 4 meses por $68,9 \%$ dos entrevistados, entre 4 e 6 meses por 21,3\%, e em menos de 2 meses por $9,8 \%$. Exercícios com a bola sem contato com outros atletas foram liberados após um período de 4 a 6 meses por $72,1 \%$ dos médicos; 16,4\% optaram pela liberação em 6 a 8 meses; 9,8\%, em 2 a 4 meses; e apenas 1,6\%, em 8 a 10 meses. Ninguém optou por mais de dez meses de restrição.

O retorno ao esporte sem restrições foi liberado por $65,6 \%$ dos entrevistados entre 6 e 8 meses de pós-operatório; $24,6 \%$ liberam o retorno entre 8 e 10 meses; 8,2\%, entre 4 e 6 meses; e apenas $1,6 \%$ depois de mais de 10 meses. Nenhum entrevistado optou por liberação em menos de quatro meses. 0 principal parâmetro usado foi o teste de força isocinético (49,2\%). 0 segundo critério mais usado foi o tempo de reabilitação e pósoperatório maior do que 6 meses (23\%), seguido do exame físico

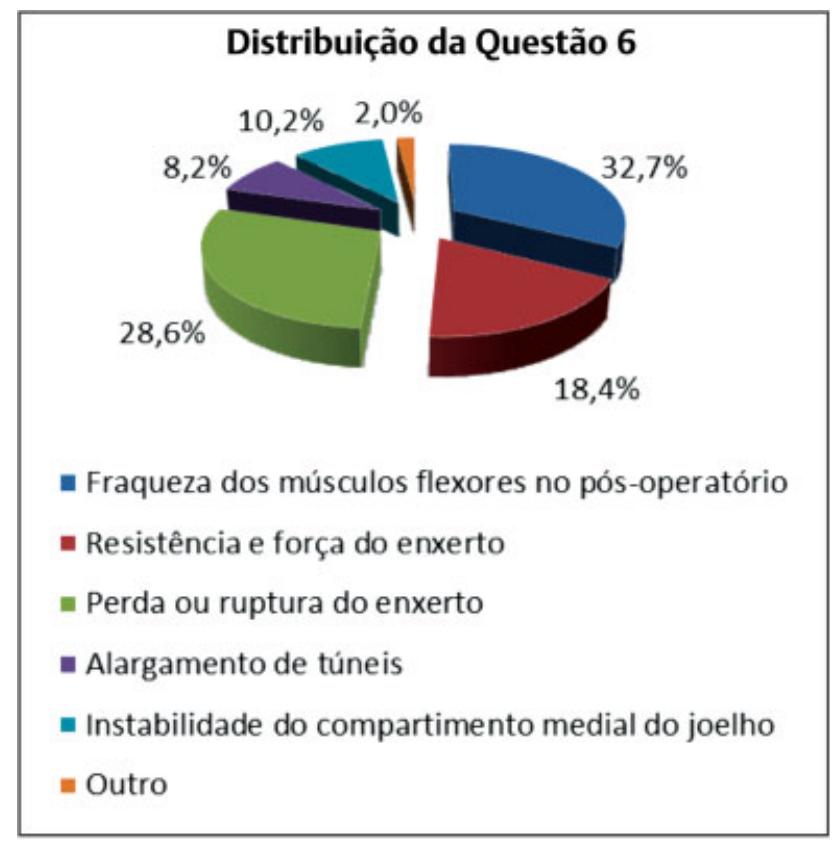

Fig. 1 Distribuição das respostas obtidas na questão 6. Estatisticamente, não houve diferença entre a resposta mais recorrente ("fraqueza dos músculos flexores no pós-operatório") e as respostas "perda ou ruptura do enxerto" $(p=0,661)$ e "resistência e força do enxerto" $(p=0,105)$.

\section{Distribuição da Questão 5}

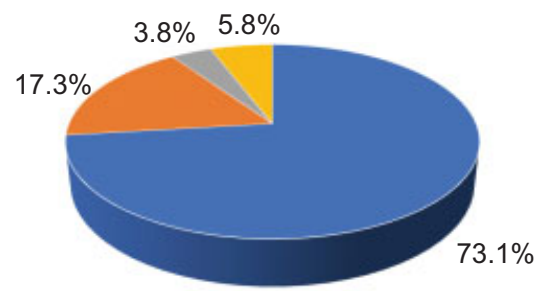

- Dor na região anterior no joelho

- Fraqueza do quadríceps por lesão ao mecanismo extensor

- Falha do enxerto - ruptura ou perda da fixação

Outra

Fig. 2 Distribuição das respostas obtidas na questão 5 . A maior preocupação consistiu na dor na região anterior do joelho. Entre as

normal e indolor (13,1\%), do teste de salto (hop test, $9,8 \%$ ), da avaliação fisioterápica (8,2\%), e de outros parâmetros (8,2\%), entre eles a combinação dos parâmetros citados anteriormente com cinemática e plataforma de força, testes funcionais 
comparativos ao lado contralateral, e aplicação do "Functional Movement Screen".

O uso de órteses funcionais é recomendado por apenas $9,8 \%$ dos especialistas. Entre aqueles que recomendaram seu uso, 83,3\% mantêm as órteses por 2 semanas após a cirurgia, enquanto $16,7 \%$ as mantêm por 3 semanas.

Quanto à porcentagem de atletas profissionais de futebol que voltam a jogar profissionalmente após a reconstrução do LCA, 73,8\% dos médicos estimam que mais de $90 \%$ retornam ao nível profissional, $18 \%$ acreditam que cerca de $80-90 \%$ retornam, e $8,2 \%$ acreditam que $60-80 \%$ retornam ao futebol profissional. A - Figura 3 descreve as respostas obtidas quanto ao retorno ao nível prévio (ou maior) de desempenho quando comparado ao nível pré-lesão.

A - Figura 4 descreve a distribuição das respostas referentes à média de reconstruções do LCA realizadas por ano pelos especialistas, enquanto a - Figura 5 descreve os resultados relacionados à média de reconstruções do LCA realizadas por ano especificamente em jogadores de futebol de todos os níveis esportivos.

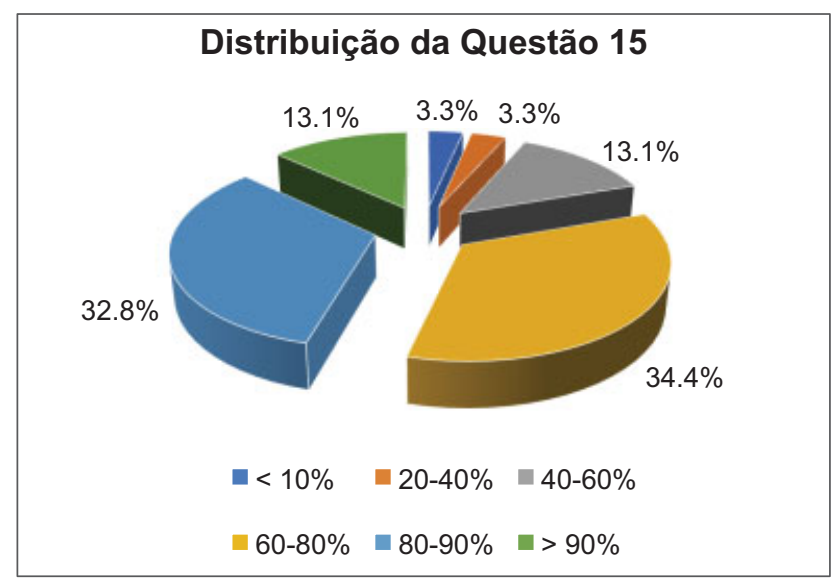

Fig. 3 Distribuição das respostas obtidas na questão 15. Não houve diferença estatística entre as opções " $80-90 \%$ " (mais frequente) e "60$80 \% "(p=0,848)$.

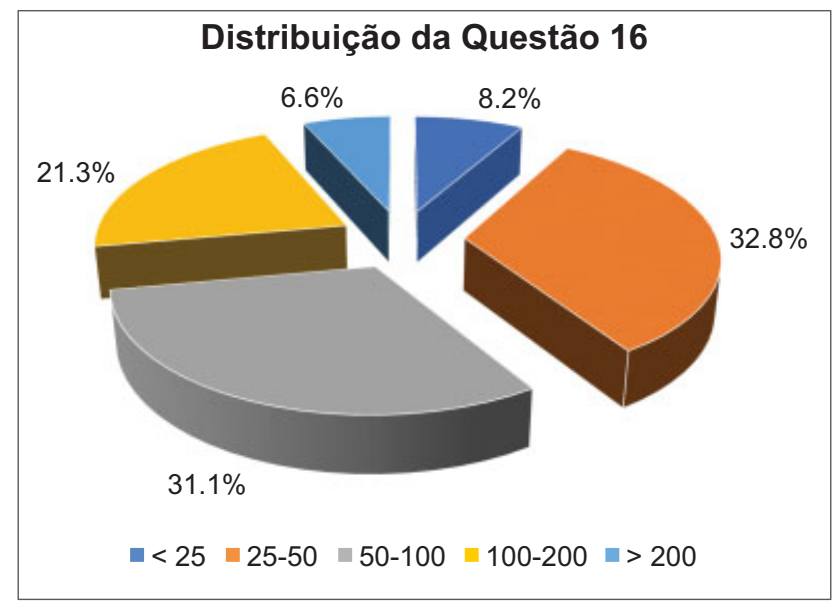

Fig. 4 Média de reconstruções. As respostas envolvendo os valores "25-50", "50-100" e "100-200" foram estatisticamente iguais $(p=0,846$ e $p=0,154)$.

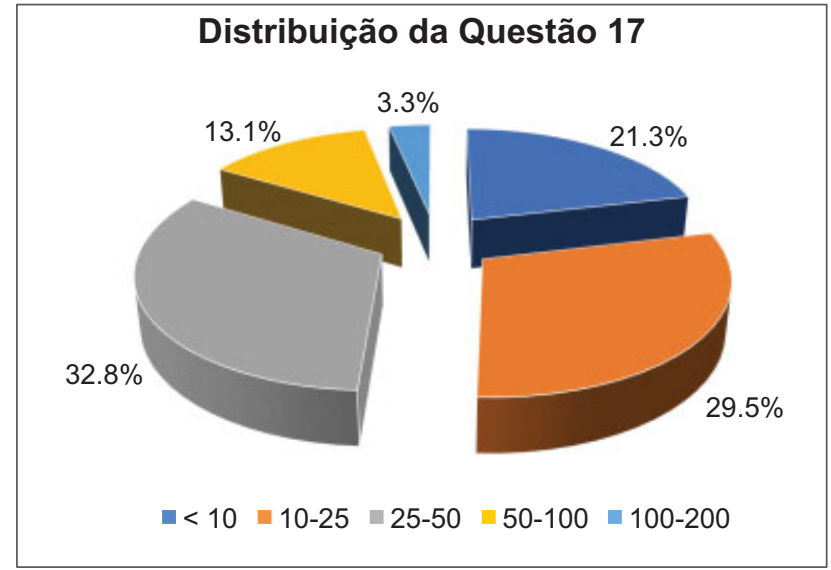

Fig. 5 Média de reconstruções do LCA realizadas por ano especificamente em jogadores de futebol. Estatisticamente, as respostas " $25-50$ ", “ $10-25$ " e “<10" não apresentaram diferenças $(p=0,696$ e $p=0,154)$.

\section{Discussão}

O sistema de divisões do Campeonato Brasileiro de Futebol é baseado na qualidade técnica dos times e no desempenho das equipes no ano anterior. A série $A$ é a primeira divisão do campeonato, e está associada aos clubes com melhor desempenho; na sequência estão as séries $B, C$ e $D$, respectivamente. Neste estudo, a maioria dos entrevistados está vinculada a clubes da série A, e embora no estudo haja representantes de todas as séries, ele envolve predominantemente ortopedistas que operam atletas do mais alto nível técnico nacional. De acordo com o nosso conhecimento, não há outros estudos similares na literatura brasileira envolvendo tantos ortopedistas e traumatologistas especializados na área, e, ao revisar a literatura, encontramos apenas três estudos similares. ${ }^{4,13,14}$

Os entrevistados são predominantemente médicos de meia idade, formados há muito tempo, e com grande experiência ortopédica e no futebol. Em sua maioria, optam por esperar entre uma e quatro semanas para o tratamento cirúrgico definitivo após a lesão do LCA. Acreditava-se que a reconstrução tardia do LCA levava a menor risco de rigidez e artrofibrose do joelho. ${ }^{15,16}$ Entretanto, estudos mais recentes relatam que a reconstrução aguda dessas lesões é segura, e não aumenta o risco de rigidez do joelho. ${ }^{15}$ Segundo Marcacci et al ${ }^{17}$, pacientes com reconstrução aguda do LCA retornaram às atividades esportivas mais rapidamente e com melhores resultados clínicos.

Os entrevistados demonstraram preferência pela técnica de reconstrução do LCA com banda única por via artroscópica. Trata-se de tópico controverso, embora não tenham sido descritas diferenças significativas em múltiplas revisões sistemáticas e metanálises na avaliação pós-operatória de pacientes que realizaram as duas técnicas. ${ }^{18-20}$

Nenhum entrevistado optou pelo uso de aloenxertos para a reconstrução do LCA. A ausência da disponibilidade e o pequeno número de biobancos no Brasil tornam esse achado previsível, e estudos da literatura estrangeira estão de acordo. Farber et $\mathrm{al}^{4}$ indicam que os aloenxertos não foram a primeira opção de nenhum cirurgião, e a maioria dos 
ortopedistas acredita que a chance de falha dos aloenxertos é maior. De fato, a taxa de nova lesão do LCA após reconstrução com aloenxerto é entre quatro e oito vezes maior em relação à reconstrução com autoenxerto em atletas e militares. ${ }^{3,4}$ Não há diferenças entre aloenxertos ou autoenxertos na qualidade do reparo de lesões do LCA. ${ }^{21}$

O método preferido para a perfuração do túnel femoral foi o portal acessório medial, seguido pela técnica outside-in, e a transtibial. Sabe-se que este é mais um tópico controverso na literatura, conforme ilustrado por Luzo et $\mathrm{al}^{22}$ e por Farber et $\mathrm{al}^{4}$ Múltiplos estudos biomecânicos ${ }^{23,24}$ demonstraram que o túnel femoral realizado via portal acessório medial cobre uma maior parte do footprint do LCA no fêmur do que o túnel por via transtibial. No entanto, faltam desfechos significativos e clinicamente relevantes. ${ }^{7,25}$

O tendão patelar é considerado a escolha ideal de enxerto, embora problemas como perda de sensibilidade, fratura da patela, contratura patelar inferior e perda do torque de extensão tenham sido relatados após a retirada dos enxertos. A principal preocupação associada ao autoenxerto do tendão patelar foi dor na região anterior do joelho, queixa comum e limitante associada a essa técnica cirúrgica. ${ }^{26} 0$ uso de autoenxertos de tendões flexores vem crescendo em popularidade porque muitos relatos sugerem que seu uso acarreta um número menor de complicações locais. Fraqueza dos músculos flexores no pós-operatório, resistência e força do enxerto, e perda ou ruptura do enxerto foram as preocupações mais citadas, também de acordo com os achados na literatura. $^{15,26}$

As taxas de retorno à atividade esportiva e função de joelho não são significativamente diferentes entre os dois grupos de enxertos mais usados, ${ }^{27}$ embora haja poucos estudos bem desenhados e randomizados comparando os métodos. ${ }^{26} \mathrm{O}$ retorno às competições foi liberado entre seis e oito meses predominantemente sem o uso de órteses, conforme relatado em outros estudos. ${ }^{4,7,15}$ Embora o estudo isocinético tenha sido o principal parâmetro usado para liberação de retorno ao esporte, vale ressaltar que o questionário não detalhou qual aparelho foi utilizado nem qual o protocolo aplicado, o que acreditamos ser um ponto importante para estudos futuros.

A maior parte dos ortopedistas acredita que os jogadores de futebol retornam com sucesso ao esporte e aos níveis esportivos pré-lesão. Uma revisão sistemática realizada por Mohtadi et al $^{28}$ demonstrou taxa de retorno ao esporte entre $63 \%$ e $97 \%$ para atletas altamente competitivos, valor compatível com os achados de Zaffagnini et al $^{12}$ (62 a 95\%). 0 retorno ao esporte no futebol profissional europeu é bastante alto, com 97\% dos atletas da Liga dos Campeões da União das Associações Europeias de Futebol (Union of European Football Associations, UEFA) retornando aos mesmos níveis anteriores à lesão após reconstrução do LCA. No entanto, apenas $65 \%$ desses atletas continuam a jogar no mesmo nível esportivo após três anos. ${ }^{29}$

Embora este seja um estudo com nível V de evidência, a opinião de múltiplos especialistas com volume cirúrgico variável, chegando a mais de 200 reconstruções de LCA por ano, revelou informações importantes. Devemos, entretanto, nos lembrar dos vieses inerentes ao uso de questionários em estudos científicos. Outros fatores a serem considerados são o predomínio do sexo masculino no esporte no país, a desconsideração de lesões ou doenças associadas, e a ausência do detalhamento de outros aspectos cirúrgicos técnicos, como método de fixação do enxerto, instrumental, uso de adjuvantes, entre outros.

\section{Conclusão}

Os especialistas em ortopedia e traumatologia associados a grandes clubes de futebol no Brasil aguardam entre uma e quatro semanas após a lesão do LCA para realizar o tratamento cirúrgico. Utilizam preferencialmente técnica com incisão e banda únicas auxiliada por artroscopia, perfuração do túnel femoral via portal acessório medial, e uso de autoenxerto quadruplo de tendões flexores ou autoenxerto do tendão patelar.

Os jogadores são liberados para retornar ao esporte sem restrições após seis a oito meses da cirurgia. 0 parâmetro usado para liberação e retorno ao esporte é o teste de força isocinético, e eles não utilizam órteses funcionais no pósoperatório.

Segundo os entrevistados, mais de $90 \%$ dos jogadores operados por lesão do LCA retornam ao esporte profissional, sendo que entre $60 \%$ e $90 \%$ retornam ao mesmo nível ou com melhor desempenho.

Portanto, o presente estudo descreve de forma satisfatória as principais práticas cirúrgicas e pós-operatórias adotadas pelos especialistas nessa população altamente específica de pacientes.

Conflitos de Interesse

Os autores declaram não haver conflitos de interesse.

\section{Referências}

1 Fédération Internationale de Football Association. FIFA Big Count 2006. Disponivel em: http://www.fifa.com/mm/document/fifafacts/bcoffs urv/bigcount.statspackage_7024.pdf

2 Astur DC, Xerez M, Rozas J, Debieux PV, Franciozi CE, Cohen M. Lesões do ligamento cruzado anterior e do menisco no esporte: incidência, tempo de prática até a lesão e limitações causadas pelo trauma. Rev Bras Ortop 2016;51(06):652-656

3 Inklaar H. Soccer injuries. I: Incidence and severity. Sports Med 1994;18(01):55-73

4 Farber J, Harris JD, Kolstad K, McCulloch PC. Treatment of Anterior Cruciate Ligament Injuries by Major League Soccer Team Physicians. Orthop J Sports Med 2014;2(11):2325967114559892. Doi: $10.1177 / 2325967114559892$

5 Brito J, Soares J, Rebelo AN. Prevenção de lesões do ligamento cruzado anterior em futebolistas. Rev Bras Med Esporte 2009;15(08):62-69

6 Waldén M, Hägglund M, Magnusson H, Ekstrand J. Anterior cruciate ligament injury in elite football: a prospective three-cohort study. Knee Surg Sports Traumatol Arthrosc 2011;19(01):11-19

7 Arliani GG, Astur DC, Kanas M, Kaleka CC, Cohen M. Lesão do ligamento cruzado anterior: tratamento e reabilitação. Perspectivas e tendências atuais. Rev Bras Ortop 2012;47(02):191-196

8 Gali JC, Mod MSB, Mimura HM, Kushiyama W. Reconstrução anatômica do ligamento cruzado anterior com dupla banda: estudo prospectivo com seguimento de dois anos. Rev Bras Ortop 2011;46(01):31-36 
9 Waldén M, Hägglund M, Werner J, Ekstrand J. The epidemiology of anterior cruciate ligament injury in football (soccer): a review of the literature from a gender-related perspective. Knee Surg Sports Traumatol Arthrosc 2011;19(01):3-10

10 Roi G, Nanni G, Tencone F. Time to return to professional soccer matches after ACL reconstruction. Sport Sci Health 2006;1(04): 142-145

11 Shelbourne KD, Gray T. Anterior cruciate ligament reconstruction with autogenous patellar tendon graft followed by accelerated rehabilitation. A two- to nine-year followup. Am J Sports Med 1997;25(06):786-795

12 Zaffagnini S, Grassi A, Marcheggiani Muccioli GM, et al. Return to sport after anterior cruciate ligament reconstruction in professional soccer players. Knee 2014;21(03):731-735

13 Erickson BJ, Harris JD, Fillingham YA, et al. Anterior cruciate ligament reconstruction practice patterns by NFL and NCAA football team physicians. Arthroscopy 2014;30(06):731-738

14 Bradley JP, Klimkiewicz JJ, Rytel MJ, Powell JW. Anterior cruciate ligament injuries in the National Football League: epidemiology and current treatment trends among team physicians. Arthroscopy 2002;18(05):502-509

15 Marder RA, Raskind JR, Carroll M. Prospective evaluation of arthroscopically assisted anterior cruciate ligament reconstruction. Patellar tendon versus semitendinosus and gracilis tendons. Am J Sports Med 1991;19(05):478-484

16 Shelbourne KD, Wilckens JH, Mollabashy A, DeCarlo M. Arthrofibrosis in acute anterior cruciate ligament reconstruction. The effect of timing of reconstruction and rehabilitation. Am J Sports Med 1991;19(04):332-336

17 Marcacci M, Zaffagnini S, Iacono F, Neri MP, Petitto A. Early versus late reconstruction for anterior cruciate ligament rupture. Results after five years of followup. Am J Sports Med 1995;23(06):690-693

18 Desai N, Björnsson H, Musahl V, et al. Anatomic single- versus double-bundle ACL reconstruction: a meta-analysis. Knee Surg Sports Traumatol Arthrosc 2014;22(05):1009-1023

19 Tiamklang T, Sumanont S, Foocharoen T, Laopaiboon M. Doublebundle versus single-bundle reconstruction for anterior cruciate ligament rupture in adults. Cochrane Database Syst Rev 2012;11: CD008413
$20 \mathrm{Xu} \mathrm{M}$, Gao S, Zeng C, et al. Outcomes of anterior cruciate ligament reconstruction using single-bundle versus double-bundle technique: meta-analysis of 19 randomized controlled trials. Arthroscopy 2013;29(02):357-365

21 Jia YH, Sun PF. Comparison of Clinical Outcome of Autograft and Allograft Reconstruction for Anterior Cruciate Ligament Tears. Chin Med J (Engl) 2015;128(23):3163-3166

22 Luzo MVM, Franciozi CE, Rezende FC, Gracitelli GC, Debieux PV, Cohen M. Ligamento cruzado anterior. Rev Brasil Ortop 2016;51 (04):385-395

23 Gadikota HR, Sim JA, Hosseini A, Gill TJ, Li G. The relationship between femoral tunnels created by the transtibial, anteromedial portal, and outside-in techniques and the anterior cruciate ligament footprint. Am J Sports Med 2012;40(04):882-888

24 Robert HE, Bouguennec N, Vogeli D, Berton E, Bowen M. Coverage of the anterior cruciate ligament femoral footprint using 3 different approaches in single-bundle reconstruction: a cadaveric study analyzed by 3-dimensional computed tomography. Am J Sports Med 2013;41(10):2375-2383

25 Chalmers PN, Mall NA, Cole BJ, Verma NN, Bush-Joseph CA, Bach BR Jr. Anteromedial versus transtibial tunnel drilling in anterior cruciate ligament reconstructions: a systematic review. Arthroscopy 2013;29(07):1235-1242

26 Aune AK, Holm I, Risberg MA, Jensen HK, Steen H. Four-strand hamstring tendon autograft compared with patellar tendon-bone autograft for anterior cruciate ligament reconstruction. A randomized study with two-year follow-up. Am J Sports Med 2001;29 (06):722-728

27 Cohen M, Ferretti M, Amaro JT. Reconstrução do ligamento cruzado anterior: escolha do enxerto. Projeto Diretrizes. São Paulo: Associação Médica Brasileira; Conselho Federal de Medicina, Sociedade Brasileira de Ortopedia e Traumatologia, São Paulo; 2007

28 Mohtadi NG, Chan DS. Return to Sport-Specific Performance After Primary Anterior Cruciate Ligament Reconstruction: A Systematic Review. Am J Sports Med 2017; •*0:363546517732541

29 Arundale AJH, Silvers-Granelli HJ, Snyder-Mackler L. Career length and injury incidence after anterior cruciate ligament reconstruction in major league soccer players. Orthop J Sports Med 2018;6(01):2325967117750825 